\title{
ATUAÇÃO DA ATENÇÃO BÁSICA NA PREVENÇÃO DO ABUSO SEXUAL INFANTIL
}

\section{REVISÃO INTEGRATIVA}

ROCHA, Keila Almeida da ${ }^{1}$, SILVA, Aline dos Santos ${ }^{2}$, SILVA, Jardeane Santos ${ }^{3}$, LEITE, Cristina Limeira ${ }^{4}$

ROCHA, Keila Almeida da. Atuação da atenção básica na prevenção do abuso sexual infantil. Revista Científica Multidisciplinar Núcleo do Conhecimento. Ano 06, Ed. 09, Vol. 04, pp. 162-175. Setembro de 2021. ISSN: 2448-0959, Link de acesso: https://www.nucleodoconhecimento.com.br/saude/atencao-basica, DOI: 10.32749/nucleodoconhecimento.com.br/saude/atencao-basica

\section{RESUMO}

Introdução: Estima-se, que por ano, cerca de 40 milhões de crianças e adolescentes sofram abuso sexual. Entre os anos de 2015 e 2016 houve cerca de 37 mil denúncias de abuso sexual com vítimas com idade entre zero a 18 anos. Pergunta norteadora: Quais as estratégias utilizadas pela atenção para a prevenção do abuso sexual infantil? Objetivo: O objetivo deste estudo foi descrever as estratégias utilizadas da atenção básica na prevenção do abuso sexual infantil. Métodos: Tratase, de uma revisão de literatura integrativa realizado por meio de levantamento bibliográfico, de natureza quantitativo. Resultados: As estratégias mais utilizadas pela atenção básica na prevenção do abuso sexual infantil são: adoção e melhoria de políticas públicas, dispositivos legais como Programa Nacional de Atenção Integral a Saúde da Criança, Programa Saúde na Escola, projetos e ações voltadas para a família e comunidade. Os presentes resultados corroboraram com achados anteriores, nos quais endossam a discussão em ambiente escolar e 0 atendimento

\footnotetext{
${ }^{1}$ Discente do curso de Enfermagem da Universidade Ceuma - Campus Imperatriz/ MA.

${ }^{2}$ Discente do curso de Enfermagem da Universidade Ceuma - Campus Imperatriz/ MA.

${ }^{3}$ Discente do curso de Enfermagem da Universidade Ceuma - Campus Imperatriz/ MA.

${ }^{4}$ Bacharel em Enfermagem - Doutoranda em enfermagem e Biociências -UNIRIO/UFRJ e Mestre em Ciências Ambientais e Saúde PUC/GO.
} 
em unidade básica. Conclusões: Abuso sexual é um problema de saúde pública, e a atenção básica de saúde tem papel fundamental na prevenção. É sabido por todos que a não prevenção e/ou o acometimento causar consequências biopsicossociais para a criança e repercute na vida adulta. Necessita, portanto, de adoção de estratégias eficazes na sua prevenção.

Palavras-chave: Abuso sexual infantil, Atenção primária, Assistência de Enfermagem, Prevenção.

\section{INTRODUÇÃO}

Abuso sexual infantil trata-se de qualquer ato, que uma pessoa cometer a uma criança, de zero a 9 anos, na intenção de obter satisfação sexual, sendo os autores do abuso aqueles que tenham desenvolvimento psicossexual mais avançado que a criança, sendo através da coação ou motivação a participarem de práticas tais como: obrigar a ver cenas sexuais ou pornográficas, realizar masturbação em público, posar para fotos e/ou outros, realizar toques e caricias sexuais e as diversas práticas sexuais. Em muitos casos esses abusos iniciam-se de forma não invasiva por meio de uma sedução e sem contato físico, que são percebidos pela criança como afetos, o que facilita ao agressor usar da inocência para realização do ato (FLORENTINO, 2015; FRANÇA, 2017).

O abuso sexual está entre os mais variados tipos de violência em nível mundial. Aproximadamente 40 milhões de crianças e adolescentes, anualmente, sofram abuso sexual. Entre os anos de 2015 e 2016 houve cerca de 37 mil denúncias de abuso sexual com vítimas com idade entre zero a 18 anos. No período de 2011 a 2017 segundo dados do Ministério da Saúde (MS), foram notificados 58.037 casos de violência sexual contra crianças, em 2018 foram 32.000 vítimas de abuso, em sua maioria ocorridas dentro de casa e cometidos pelos próprios familiares, o que dificulta a denúncia e caracterização, pois quem deveria proteger, comete 0 ato (KRINDGES; MACEDO; HABIGZANG, 2016; ARAÚJO et al., 2019). 
A atenção básica, constitui um espaço primário para o atendimento, identificação, cuidados, proteção de crianças e outros em situação de violência, atuando através de programas e ações, sendo um destes o Programa Saúde na Escola. Este, por sua vez, exerce um relevante papel na prevenção de violências, por meio de ações conjunta áreas da educação e outros setores sociais. Com ênfase na prevenção de danos a saúde e a promoção do bem-estar das famílias. Dentro da atenção primária, é importante destacar o papel dos profissionais da enfermagem como linha de frente para organização e promoção de ações que implicam na prevenção dos abusos em questão (HORONATO et al., 2018; DELZIOVO et al., 2018).

O abuso sexual contra menores, representa um problema social, de saúde e de segurança pública. Sendo relevante à sua análise, intervenção e prevenção, tendo em vista as consequências traumáticas para o desenvolvimento psíquico, social e físico das vítimas. Este trabalho, tem como objetivo: descrever as estratégias que a atenção básica utiliza para a prevenção do abuso sexual infantil.

\section{METODOLOGIA}

Este estudo configura-se como revisão integrativa realizado através da coleta de dados, a partir de fontes secundárias por meio de levantamento bibliográfico. Este método de revisão busca sintetizar resultados de estudos primários, na qual a pesquisa permite realizar a síntese destes estudos e conclusões gerais acerca do tema abordado (MENDES; SILVEIRA; GALVÃO, 2018). Para norteamento da revisão integrativa, formulou-se a seguinte questão: quais as estratégias que a atenção básica utiliza para a prevenção do abuso sexual infantil?

Para o levantamento dos artigos, realizou-se uma busca nas seguintes bases de dados: Literatura Latino-Americana e do Caribe em Ciências da Saúde (LILACS) e Medical Literature Analysis and Retrieval System Online (MEDLINE). Foram utilizados os seguintes descritores no DeCS (Descritores em Ciências de Saúde) e $\mathrm{MeSH}$ (Medical Subject Headings) e suas combinações nas línguas portuguesa e inglesa: Abuso sexual na infância (Child abuse, sexual); Atenção Primária (Primary 
Care); Cuidados de Enfermagem (Nursing Care); Estratégia (Strategy); Prevenção (Prevention).

A coleta da amostra para o estudo ocorreu no período de fevereiro a abril de 2021 . Os critérios de inclusão definidos para a seleção dos artigos foram: artigos em português, inglês e espanhol, com publicação a partir de 2015. Os critérios de exclusão dos artigos foram: artigos indexados repetidamente e que não atendam ao objetivo do estudo.

\section{RESULTADOS}

Os artigos encontrados, forma selecionados por meio da leitura dos resumos, levado em consideração os critérios estabelecidos na amostra. Após a análise, os artigos copiados e salvos em formato digital PDF. Todos os artigos selecionados foram lidos integralmente. Sendo que destes, quatorze foram tomados como objeto de estudo, para identificação e registro das estratégias de atuação da atenção básica utilizadas para a prevenção do abuso sexual infantil.

A pesquisa foi obtida através das combinações dos descritores. Na base de dados, identificou-se, 14.641 artigos que foram avaliados e exclusos, nos quais não condiziam com os critérios de inclusão estabelecidos, restando 105, destes 79 identificados foram excluídos na revisão do resumo, por não descrever o tema abordado. Após a revisão de resumo, 29 artigos foram selecionados (19 artigos encontrados na base de dados da LILACS e 6 na base de dados MEDLINE), os mesmos selecionados para leitura do texto completo após a leitura 13 foram excluídos por não apresentarem aspectos que respondiam à questão norteadora desta revisão. As etapas desta avaliação estão descritas na Figura 1. 
Figura 1. Seleção de artigos

14.641 títulos identificados a parte da pesquisa na base de dados

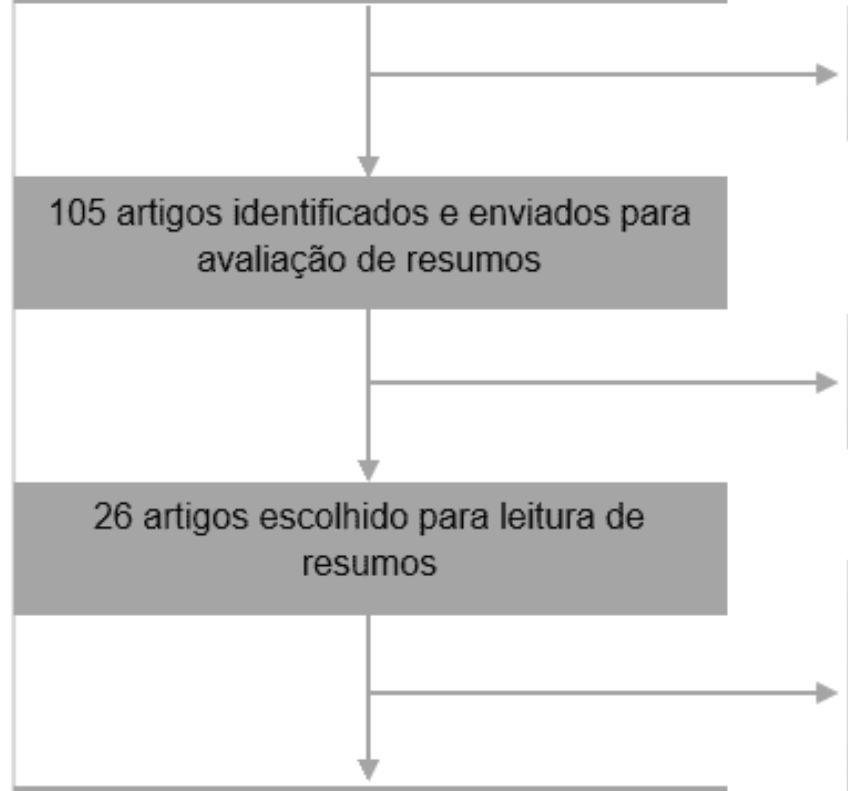

26 artigos selecionados para leitura do texto completo

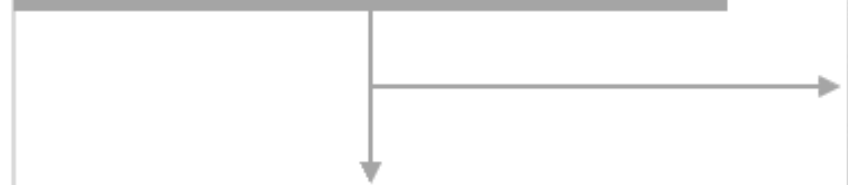

13 artigos incluídos para amostra final
14.536 excluídos (não atendia aos critérios de incluídos para amostra)

79 excluídos na leitura do resumo, por não descrever o tema abordado

19 artigos foram encontrados na base de dados da LILACS

6 artigos na base de dados MEDLINE

13 artigos foram excluídos após leitura do texto completo (não abordava atuação da atenção básica na prevenção do abuso sexual infantil)

Fonte: Elaborado pelos autores (2021).

O resultado foi composto por treze artigos científicos. Destes, dez forma encontrados na base de dados da biblioteca virtual LILACS, três na MEDLINE. (Tabela 1).

RC: 97340

Disponível em: https://www.nucleodoconhecimento.com.br/saude/atencao-basica 
Tabela 1. Artigos encontrados nas bases de dados LILACS e MEDLINE sobre as estratégias que a atenção básica utiliza para a prevenção do abuso sexual infantil.

\begin{tabular}{|c|c|c|c|c|}
\hline Ano & Periódico & Autores & Título & $\begin{array}{l}\text { Base } \\
\text { dados }\end{array}$ \\
\hline 2019 & $\begin{array}{l}\text { Psicologia: } \\
\text { Teoria } \\
\text { Prática. }\end{array}$ & $\begin{array}{l}\text { Soma SMP, } \\
\text { Williams LCA. }\end{array}$ & $\begin{array}{l}\text { Livro infantil } \\
\text { especializado como } \\
\text { estratégia de prevenção } \\
\text { do abuso sexual. }\end{array}$ & LILACS \\
\hline 2019 & $\begin{array}{l}\text { Rev. Min. } \\
\text { Enferm. }\end{array}$ & $\begin{array}{l}\text { Vieira DS, Dias } \\
\text { TKC, Pedrosa } \\
\text { RKB, Vaz EMC, } \\
\text { Collet N, Reichert } \\
\text { APS. }\end{array}$ & $\begin{array}{l}\text { Processo de trabalho de } \\
\text { enfermeiros na vigilância } \\
\text { do desenvolvimento } \\
\text { infantil. }\end{array}$ & MEDLINE \\
\hline 2018 & $\begin{array}{l}\text { Rev. Latino-Am. } \\
\text { Enferm. }\end{array}$ & $\begin{array}{l}\text { Yakuwa MS, Neill } \\
\text { S, Mello DF. }\end{array}$ & $\begin{array}{llr}\text { Estratégias } & & \text { de } \\
\text { enfermeiros } & \text { para } & \text { a } \\
\text { vigilância à saúde } & \text { da } \\
\text { criança. } & & \end{array}$ & LILACS \\
\hline 2018 & $\begin{array}{l}\text { Rev. Enferm. } \\
\text { do } \quad \text { Centro- } \\
\text { Oeste Mineiro. }\end{array}$ & $\begin{array}{l}\text { Branquinho ID, } \\
\text { Lanza FM. }\end{array}$ & $\begin{array}{l}\text { Saúde da criança na } \\
\text { atenção primária: } \\
\text { evolução das políticas } \\
\text { brasileiras e a atuação do } \\
\text { enfermeiro. }\end{array}$ & LILACS \\
\hline 2018 & $\begin{array}{l}\text { Psiquiatría Y } \\
\text { Salud Mental. }\end{array}$ & 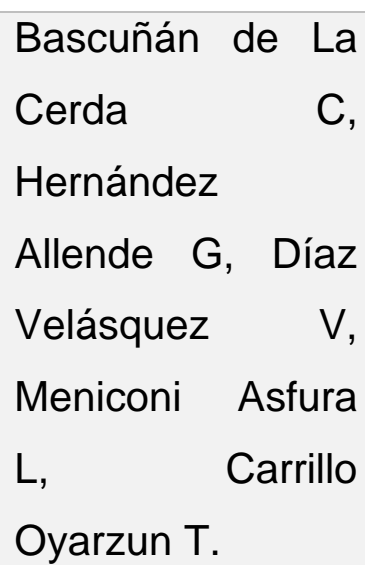 & $\begin{array}{l}\text { Proyecto de promoción } \\
\text { de Salud "Mi Tesoro": El } \\
\text { abuso sexual infantil o } \\
\text { infanto-juvenil (ASI). }\end{array}$ & LILACS \\
\hline
\end{tabular}


2018 Primary Health Jones D, Ballard A community engaged MEDLINE Care Research J, Dyson R, primary healthcare \& Development. Macbeth $\mathrm{P}$, Lyle strategy to address rural $D$, Sunny $P$, school student inequities: Thomas A, a descriptive paper.

Sharma I.

2018 Rev. Saúde Wald G, Camarotti Modelo comunitario para MEDLINE Coletiva. AC, Capriati AJ, la promoción, prevención, kornblit AL. asistencia y protección ante situaciones de abuso sexual contra niñas, niños $\quad y$ adolescentes.

2017 Rev. Paul. Leoncio ET, Degradação do vínculo LILACS Pediatr. Souza SRP, parental e violência Machado JLM. contra a criança: o uso do genograma familiar na prática clínica pediátrica.

\begin{tabular}{|c|c|c|c|c|}
\hline 2017 & $\begin{array}{l}\text { Pesq. Prátic. } \\
\text { Psicoss. }\end{array}$ & França CP. & $\begin{array}{l}\text { Um corpo maculado na } \\
\text { infância: a necessidade } \\
\text { de intervenção precoce } \\
\text { dos profissionais de } \\
\text { saúde. }\end{array}$ & LILACS \\
\hline 2017 & Trends Psychol. & $\begin{array}{l}\text { Soma SMP, } \\
\text { Williams LCA. }\end{array}$ & $\begin{array}{l}\text { Avaliação de Livros } \\
\text { Infantis Brasileiros sobre } \\
\text { Prevenção de Abuso } \\
\text { Sexual baseada em } \\
\text { Critérios da Literatura. }\end{array}$ & LILACS \\
\hline 2016 & $\begin{array}{l}\text { Ver. Gaúcha } \\
\text { Enferm. }\end{array}$ & $\begin{array}{lr}\text { Leite } & \text { JT, Beserra } \\
\text { MA, Scatena L, } \\
\text { Silva }\end{array}$ & $\begin{array}{l}\text { Enfrentamento da } \\
\text { violência doméstica } \\
\text { contra crianças e }\end{array}$ & LILACS \\
\hline
\end{tabular}




\begin{tabular}{|c|c|c|c|c|}
\hline & & Ferriani MGC. & $\begin{array}{l}\text { adolescentes na } \\
\text { perspectiva } \\
\text { enfermeiros da atenção } \\
\text { básica. }\end{array}$ & \\
\hline 2016 & $\begin{array}{l}\text { Rev. Saúde } \\
\text { Coletiva. }\end{array}$ & $\begin{array}{l}\text { Deslandes SF, } \\
\text { Nascimento AF. }\end{array}$ & $\begin{array}{l}\text { A construção da agenda } \\
\text { pública brasileira de } \\
\text { enfrentamento da } \\
\text { violência sexual infanto- } \\
\text { juvenil. }\end{array}$ & LILACS \\
\hline 2015 & $\begin{array}{l}\text { Journal of } \\
\text { Human Growth } \\
\text { and } \\
\text { Development. }\end{array}$ & $\begin{array}{ll}\text { Silva } & \text { DI, } \\
\text { Veríssimo } & \text { MR, } \\
\text { Mazza VA. } & \end{array}$ & $\begin{array}{l}\text { Vulnerabilidade no } \\
\text { desenvolvimento infantil: } \\
\text { influência das políticas } \\
\text { públicas e programas de } \\
\text { saúde. }\end{array}$ & LILACS \\
\hline
\end{tabular}

Fonte: Elaborado pelos autores (2021)

\section{DISCUSSÃO}

Segundo os autores, durante muitos anos a violência doméstica assim também como a violência sexual foi problema das áreas sociais e jurídica. Nesse período os profissionais da saúde não trabalhavam a prevenção e muito menos a detecção de casos de violência sexual contra a criança, pois não possuíam a liberdade de intervir nesse tipo de ocorrência. Com o passar do tempo houve a necessidade de trazer o assunto para o debate e avaliação de diversos profissionais na área da saúde. E assim foi possível obter um nível maior de conhecimento do problema e ter a possibilidade de aprofundar os resultados obtidos na busca de uma intervenção e/ou prevenção (SILVA; VERÍSSIMO; MAZZA, 2015).

Com a implantação do Estatuto da Criança e do Adolescente (ECA), é exigido atenção prioritária por parte da família, da sociedade e do Estado para que toda criança e adolescente tenha seu desenvolvimento físico, moral e psicológico protegido por todos. Frente a este contexto, a área da saúde passa a ser essencial 
para o cuidado de crianças, garantindo o direito à vida e adesão a programas, ações e projetos que garantam a promoção, proteção e recuperação da saúde através do Sistema Único de Saúde onde a Atenção Básica passa a ser a porta de entrada para os cuidados primários (FRANÇA, 2017; BASCUÑÁN DE LA CERDA et al., 2018; JONES et al., 2019; VIEIRA et al., 2019).

A atenção básica é um espaço responsável pela identificação, acolhimento, atendimento, notificação, cuidados e proteção de crianças em situações de violências, seja através do atendimento em unidades de saúde como na visita domiciliar que possibilita obter informações e observações que contribuem para a vigilância à saúde das crianças e identificação de casos, além de promover orientações às famílias e sociedade, e tem como uma das principais estratégias a comunicação. Ferramentas para a resolução de problemas, tomada de decisões e metodologias participativas, destinadas a diferentes grupos na comunidade com objetivo de desnaturar o abuso e comprometer o maior quantitativo de pessoas para sua erradicação (DELZIOVO, 2018; EGRY, 2018).

O abuso sexual infantil como um dos tipos de violência, necessita de um olhar amplo, visto que, este causa repercussões no desenvolvimento biopsicossocial da criança. A atenção básica tem como princípios e diretrizes promover o cuidado integral ao indivíduo, biopsicossocial incluído assim a prevenção de agravos à saúde deste. A atenção básica atua na saúde da criança através da orientação estabelecidas por políticas públicas e programas voltados ao cuidado em geral (WALD et al., 2018; BRANQUINHO; LANZA, 2028; YAKUMA; NEILL; MELLO, 2018).

Diante deste contexto, a violência sexual infantil é um assunto fundamental e prioritário na saúde pública, bem como o vínculo desta no ambiente escolar. Sendo responsável por desenvolver ações de prevenção primária ao abuso sexual infantil, e as ações que podem ser desenvolvidas nas escolas são amplas, desde treinamento de professores à realização de ações voltadas ao público-alvo. Uma das ferramentas, que a atenção em saúde pode promover através de programas escolares é a literatura e diálogos claros sobre essa temática que encoraja o fortalecimento da população infantil, visto que quando ocorrer 0 ato na primeira 
tentativa de abuso, tais indivíduos reconheçam e relatem para alguém próximo a eles. Esses projetos e programas, reforçam ainda a importância da parceria, entre atenção básica em uma colaboração intersetorial com educação em ambiente escolar. Visto que, o ambiente escolar possui papel importante na formação plena da cidadania das crianças e adolescentes, todavia é na escola que passam grande parte do tempo e com isso se torna o local ideal para desenvolver tais programas (WALD et al., 2018; SOMA; WILLIAMS, 2017; SOMA; WILLIAMS, 2019).

Deslandes e Nascimento (2016), faz uma análise em seu estudo sobre a construção da Agenda Pública brasileira em relação ao enfrentamento da violência sexual infanto-juvenil e destaca o Plano Nacional como política pública que teve grande importância no enfrentamento da violência sexual, mas mesmo com as lutas travadas pelo Plano Nacional direcionadas aos direitos das crianças e adolescentes, vê se a violência sexual infanto-juvenil como um problema longe de ser superado. Existe fatores limitantes para uma implantação eficaz de políticas voltadas a temática abordada, que vai desde aspectos orçamentários às estruturas institucionais que são responsáveis por sua execução (DESLANDES; NASCIMENTO, 2016).

Alguns artigos relatam dificuldades na prevenção deste tipo de abuso. Em uma pesquisa realizada em cinco Unidades de Estratégia de Saúde da Família no Estado de São Paulo, alguns enfermeiros relataram ter conhecimento sobre as políticas públicas de prevenção voltadas a este tipo de violência, porém na ESF é priorizado outras atividades. Nesse estudo é observado ainda que há uma grande dificuldade enfrentada por enfermeiros e outros profissionais dos serviços de saúde pública por se sentirem desamparados e desconhecerem procedimentos a serem tomados no combate à violência doméstica contra crianças e adolescentes. Outro fator mencionado é a dificuldade em lidar de forma efetiva com a violência doméstica devido ao alto índice de casos, e a falta de contingente onde os casos são complexos, havendo ainda uma grande carência de capacitação continuada (LEITE et al., 2016; YAKUMA; NEILL; MELLO, 2018). 
O Programa de Atenção Integral a Saúde da Criança (PAISC) é um dos instrumentos para a atuação da estratégia Saúde da Família, que visa proporcionar a criança desenvolvimento e qualidade de vida, bem como a atenção integral a criança em situações de violências, incluindo a violência sexual. Seja através da prevenção realizado por meio de ações educativas como na eliminação de seus determinantes. O enfermeiro é um agente de grande importância na atenção à saúde da criança. É notório e de grande valia seus cuidados ao indivíduo, buscando a promover saúde e bem-estar, por meio da identificação de problemas reais e potenciais a criança e a família, direcionando-as para intervenções quando necessário (BRANQUINHO; LANZA, 2018; VIEIRA et al., 2019; SILVA; VERÍSSIMO; MAZZA, 2015).

Em relação a família como relatado em um dos artigos analisados, é base da proteção e desenvolvimento da criança. Porém diversos aspectos podem favorecer ou interferir neste processo. Como por exemplo: grau de instrução da família, caráter familiar, poder econômico e a propensão para desencadear violência ou não para a criança. Importante elencar que independente de status familiar, e o meio que essa criança está inserida, a violência contra criança pode acontecer, e de formas diversas. Todavia, os fatores citados acima contribuem em uma porcentagem maior ou menor para que a violência aconteça (LEONCIO; SOUZA; MACHADO, 2017).

Quando se tem uma criança inserida num meio onde há miséria, fome, violência, instabilidade de afeto e falta de solidariedade, pode se entender que há inúmeras causas e/ou fatores de privação de sentimentos entre os envolvidos no meio, incluindo ainda a vulnerabilidade social. Por isso, promover a busca destes fatores e direcioná-los na tentativa de corrigi-los, é uma das estratégias no trabalho de prevenção de possíveis casos de violência sexual na infância (LEITE et al., 2016; SOMA; WILLIAMS, 2017; LEONCIO; SOUZA; MACHADO, 2017).

Leoncio e demais autores (2019), a atenção integral da criança ainda tem grandes desafios na busca de métodos produtivos e inovadores. Na pediatria uma das ferramentas que possibilita uma visão mais ampla da saúde da criança é o genoma, que por sua vez é um instrumento que agrega informações biopsicossocial e 
econômica da família, com objetivo de analisar presença de vínculos familiares, conflito e violência contra a criança, além de avaliar se a criança está exposta a fatores estressores (LEONCIO; SOUZA; MACHADO, 2017).

\section{CONCLUSÃO}

O abuso sexual na infância representa um problema de saúde pública, e se não tomada estratégias para a sua prevenção pode causar danos biopsicossociais à criança e gerar repercussões na vida adulta. A atenção primária a saúde como porta de entrada, tem como princípios a integralidade da assistência à saúde através da adoção de políticas públicas, programas e elaboração de ações preventivas. As equipes da atenção básica por estarem geograficamente mais próximo das famílias, por realizar acompanhamento e pelo seu envolvimento com ações de saúde coletiva e individual, tem maior possibilidade de identificar fatores de risco para violência e realizar ações que possa intervir e prevenir.

Entretanto, apesar da importância das políticas públicas, percebe-se que, ainda falta muito para se ter uma estratégia eficaz contra a violência sexual infantil a nível de atenção básica. Diante disto é possível perceber que as equipes de atenção básica necessitam de mais incentivos por parte das políticas públicas para desenvolver ações que colabore de forma direta e indireta na prevenção da violência sexual infantil, bem como o aprimoramento de políticas públicas voltada a este público, adoção de programa Saúde na Escola, educação e orientação de pais familiares/cuidadores/sociedade.

\section{REFERÊNCIAS}

ARAÚJO, G. et al. Determinantes da violência sexual infantil no estado do Paraná Brasil. Revista Espaço Saúde. 20(2), 42-54, dezembro de 2019. Disponível em: http://espacoparasaude.fpp.edu.br/index.php/espacosaude/article/download/652/pdf. Acesso em: 15/02/2021. 
BASCUÑÁN DE LA CERDA C. et al. Proyecto de promoción de Salud "Mi Tesoro": El abuso sexual infantil o infanto-juvenil (ASI). Psiquiatría Y Salud Mental. 35(3), 215-218, 2018. Disponível em: http://www.schilesaludmental.cl/2018-2/. Acesso em: 25/02/2021.

BRANQUINHO, I. D; LANZA, F. M. Saúde da criança na atenção primária: evolução das políticas brasileiras e a atuação do enfermeiro= child health in primary care: evolution of brazilian policies and nurses' performance= Salud del niño en la atención primaria: evolución de las políticas brasileñas y la acción del enfermeiro. Revista de Enfermagem do Centro-Oeste Mineiro. 8 (e2753), 1-11, 2018. Disponível em: https://pesquisa.bvsalud.org/portal/resource/pt/biblio-973258.

Acesso

em: 25/03/2021.

DELZIOVO, C. R. et al. Atenção à saúde de crianças e adolescentes em situação de violência. Florianópolis (SC): Universidade Federal de Santa Catarina, Violência e saúde, 2018.

em: https://ares.unasus.gov.br/acervo/html/ARES/13970/1/MOOC-Crianca.pdf. Acesso em: 29/03/2021.

DESLANDES, S. F; NASCIMENTO, A. F. A construção da agenda pública brasileira de enfrentamento da violência sexual infanto-juvenil. Revista Saúde Coletiva. 26(4), 1171-1191, 2016.2 Disponível em: https://www.scielosp.org/article/physis/2016.v26n4/1171-1191/. Acesso em: 02/03/2021.

EGRY, E. Y; APOSTOLICO, M. R; MORAIS, T. C. P. Notificação da violência infantil, fluxos de atenção e processo de trabalho dos profissionais da Atenção Primária em Saúde $=$ Reporting child violence, health care flows and work process of primary health care professionals. Ciências e Saúde Coletiva. 23(1), 83-92, 2018. Disponível em: https://www.scielo.br/scielo.php?script=sci_arttext\&nrm=iso\&lng=pt\&tlng=pt\&pid=S14 13-81232018000100083. Acesso em: 24/02/2021. 
FLORENTINO, B. R. B. As possíveis consequências do abuso sexual praticado contra crianças e adolescentes. Fractal Rev Psicol. 27(2),139-144, 2015. Disponível em: http://dx.doi.org/10.1590/1984-0292/805. Acesso em: 20/03/2021.

FRANÇA, C. P. Um corpo maculado na infância: a necessidade de intervenção precoce dos profissionais de saúde $=\mathrm{A}$ body sullied in childhood: the need for early intervention of health professionals $=$ Un cuerpo inmaculado en la infancia: la necesidad de intervención precoz de los profesionales de la salud. Pesq. Práticas Psicossoc. 12(3), 1-10, 2017. Disponível em: http://pepsic.bvsalud.org/scielo.php?script=sci_arttext\&pid=S180989082017000300007. Acesso em: 21/02/2021.

HOHENDORFF, J. V; PATIAS, N. D. Violência sexual contra crianças e adolescentes: identificação, consequências e indicações de manejo. BarBarói. 0(49), 239-257, 2017. Disponível em: http://dx.doi.org/10.17058/barbaroi.v0i49.9474. Acesso em: 19/02/2021.

HONORATO, L. G. F, et al. Violência na Infância e Adolescência: Perfil notificado na mesorregião do Baixo Amazonas. Arq Bras Psicol. 70:(2), 266-284, 2018. Disponível em: http://pepsic.bvsalud.org/scielo.php?script=sci_abstract\&pid=\$180952672018000200019\&Ing=pt. Acesso em: 20/02/2021.

JONES, D, et al. A community engaged primary healthcare strategy to address rural school student inequities: a descriptive paper. Primary Health Care Research \& Development. 20(26), 1-6, 2019. Disponível em: https://www.cambridge.org/core/journals/primary-health-care-research-anddevelopment/article/community-engaged-primary-healthcare-strategy-to-addressrural-school-student-inequities-a-descriptivepaper/4D76560E00A5637F9221A99E3852232C. Acesso em: 18/03/2021.

KRINDGES, C. A; MACEDO, D. M; HABIGZANG, L. F. Abuso sexual na infância e suas repercussões na satisfação sexual na idade adulta de mulheres vítimas. 
Contextos Clínic. 119(1), 60-71, 2016. Disponível em: http://dx.doi.org/10.4013/ctc.2016.91.05. Acesso em: 08/03/2021.

Leite, J. T. et al. Enfrentamento da violência doméstica contra crianças e adolescentes na perspectiva de enfermeiros da atenção básica= Coping with domestic violence against children and adolescents from the perspective of primary care nurses $=\mathrm{El}$ enfrentamiento de la violencia domestica contra niños y adolescentes bajo la perspectiva de enfermeros de la atención básica. Rev. Gaúcha Enferm. $37(2), \quad 1-7, \quad 2016 . \quad$ Disponível em: https://www.scielo.br/scielo.php?script=sci_arttext\&nrm=iso\&lng=pt\&tlng=pt\&pid=S19 83-14472016000200415. Acesso em: 30/02/2021.

LEONCIO, E. T; SOUZA, S. R. P; MACHADO, J. L. M. Degradação do vínculo parental e violência contra a criança: o uso do genograma familiar na prática clínica pediátrica $=$ Degradation of parental bonding and violence against children: the use of family genogram in the pediatric clinic. Rev. Paul. Pediatr. 35(2),185-190, 2017. Disponível em: https://www.scielo.br/pdf/rpp/2017nahead/en_0103-0582-rpp-201735-2-00009.pdf. Acesso em: 09/03/2021.

MENDES, K. D. S; SILVEIRA, R. C. C. P; GALVÃO, C. M. Revisão integrativa: método de pesquisa para a incorporação de evidências na saúde e na enfermagem= Integrative literature review: a research method to incorporate evidence in health care and nursing = Revisión integradora: método de investigación para la incorporación de evidencias en la salud y la enfermeira. Texto Contexto Enferm [Internet]. 17(4), 758-64, 2018. Disponível em: https://scielo.br/pdf/tce/v17n4/18.pdf. Acesso em: 23/02/2021.

SILVA, D. I; VERÍSSIMO, M. R; MAZZA, V. A. Vulnerabilidade no desenvolvimento infantil: influência das políticas públicas e programas de saúde= Vulnerability in the child development: influence of public policies and health programs. Journal of Human Growth and Development. 25(1),11-18, 2015. Disponível em: https://pesquisa.bvsalud.org/portal/resource/pt/lil-747941. Acesso em: 16/03/2021. 
SOMA, S. M. P; WILLIAMS. L. C. A. Avaliação de Livros Infantis Brasileiros sobre Prevenção de Abuso Sexual baseada em Critérios da Literatura. Trends Psychol. 25(3), 1201-1212, $2017 . \quad$ Disponível em: http://pepsic.bvsalud.org/scielo.php?script=sci_arttext\&pid=S1413389X2017000300014. Acesso em: 20/02/2021.

SOMA, S. M. P; WILLIAMS. L. C. A. Livro infantil especializado como estratégia de prevenção do abuso sexual. Psicologia: Teoria e Prática. 21(1), 186-203, 2019. Disponível em: http://pepsic.bvsalud.org/scielo.php?script=sci_arttext\&pid=S151636872019000100008. Acesso em: 21/02/2021.

VIEIRA, D. S. et al. Processo de trabalho de enfermeiros na vigilância do desenvolvimento infantil $=$ Work process of nurses in child development surveillance $=$ Proceso de trabajo de enfermeros en vigilancia del desarrollo infantil. Rev. Min. Enferm. 23(e1242), 1-8, 2019. Disponível em: https://cdn.publisher.gn1.link/reme.org.br/pdf/e1242.pdf. Acesso em: 23/02/2021.

WALD, G. et al. Modelo comunitario para la promoción, prevención, asistencia y protección ante situaciones de abuso sexual contra niñas, niños y adolescentes. Rev. Saúde Coletiva. 28(4), 1-25, 2018. Disponível em: https://www.scielo.br/scielo.php?script=sci_arttext\&pid=S0103-73312018000400609. Acesso em: 24/02/2021.

YAKUWA, M. S; NEILL, S; MELLO, D. F. Estratégias de enfermeiros para a vigilância à saúde da criança. Rev. Latino-Am. Enferm. 26(e3007), 1-8, 2018. Disponível em: https://www.scielo.br/scielo.php?script=sci_arttext\&nrm=iso\&lng=pt\&tlng=pt\&pid=S01 04-11692018000100329. Acesso em: 25/02/2021.

Enviado: Setembro, 2021.

Aprovado: Setembro, 2021. 